\title{
Analysis of Synchronization of Respiration-induced Motions in the Duodenum and Stomach for the Primary Tumor in Pancreatic Cancer Using Four- Dimensional Computed Tomography
}

Rei Umezawa ( $\nabla$ reirei513@hotmail.com )

Tohoku University Graduate School of Medicine https://orcid.org/0000-0002-7280-3676

Akihisa Wakita

National Cancer Center Hospital

Yoshiyuki Katsuta

Tohoku University Graduate School of Medicine

Yoshinori Ito

National Cancer Center Hospital

Satoshi Nakamura

National Cancer Center Hospital

Hiroyuki Okamoto

National Cancer Center Hospital

Noriyuki Kadoya

Tohoku University Graduate School of Medicine

Kana Takahashi

National Cancer Center Hospital

Koji Inaba

National Cancer Center Hospital

Naoya Murakami

National Cancer Center Hospital

Hiroshi Igaki

National Cancer Center Hospital

Keiichi Jingu

Tohoku University Graduate School of Medicine

Jun Itami

National Cancer Center Hospital

Research 
Keywords: Pancreatic cancer, Radiotherapy, Four-dimensional computed tomography, Organ motion.

Posted Date: July 29th, 2020

DOI: https://doi.org/10.21203/rs.3.rs-48776/v1

License: (c) (1) This work is licensed under a Creative Commons Attribution 4.0 International License. Read Full License 


\section{Abstract}

Background: We investigated the synchronization of respiration-induced motions at the primary tumor and organs at risk at radiation planning for pancreatic cancer.

Methods: Four-dimensional (4D) computed tomography images were acquired under the condition of shallow free breathing in patients with pancreatic cancer. The gross tumor volume (GTV), duodenum (DU) and stomach (ST) were contoured. With reference to the $50 \%$ phase (exhale phase), excursions of respiration-induced motions of the GTV, DU and ST were measured. Based on the shift of the GTV, we investigated the synchronization of respiration-induced motions between each contouring target. We examined the differences in the overlap volumes between PTV and the planning organ at risk volume (PRV) in ST and DU and the differences in mean doses to the ST and DU in each respiratory phase.

Results: Nine patients with pancreatic cancer were analyzed in this study. The mean maximum threedimensional excursions at the GTV, DU and ST were 9.6, 9.8 and $11.4 \mathrm{~mm}$, respectively. At phase $0 \%$ and $90 \%$ (inhale phases), mean distance changes in the positional relationship with the GTV were 0.3 and 0.7 $\mathrm{mm}$, respectively, for the DU, and -2.5 and $-2.4 \mathrm{~mm}$, respectively for the ST, respectively. There was no significant difference in distance changes between each respiratory phase in the DU $(p=0.568)$, while there was a significant difference in distance changes in the ST $(p<0.001)$. There was a slight increase in the overlap volume between PTV and PRV in the expiratory phase in ST, but there were no significant differences between the respiratory phases either in ST or DU $(p=0.101$ and 0.559$)$. There was a significant difference in the change rates of mean doses in the respiratory phases in ST $(p=0.023)$, but there was no significant difference in DU $(p=0.933)$.

Conclusions: Our results indicate that the DU but not the ST might move synchronously with GTV due to respiration.

\section{Background}

Radiotherapy (RT) is one of the treatment options for pancreatic cancer. Recently, results of studies on the effects of dose escalation for intensity-modulated radiation therapy (IMRT) and stereotactic radiation therapy (SRT) on pancreatic cancer have been reported [1-4]. However, RT for pancreatic cancer is susceptible to respiratory movement of the primary tumor and it is necessary to consider the variation of this movement in RT planning for pancreatic cancer.

Recently, a method for the reconstruction of four-dimensional computed tomography (4DCT) images acquired during free breathing has been reported [5]. There have been many reports about movement of the primary tumor determined by using $4 \mathrm{DCT}$ in RT for pancreatic cancer, and movement of the primary tumor in the cranio-caudal directions has been shown to be more variable than that in other directions [68]. Thus, respiratory movement of the primary tumor in pancreatic cancer can be understood to some extent, but there have been few reports on respiratory movements of normal organs close to the primary tumor. Moreover, there is a possibility that organs at risk (OARs) may move without interlocking with the 
primary tumor due to breathing. In that case, evaluation of the dose distribution at RT planning may not be precisely reflected. There is a possibility that the actual dose to the stomach and duodenum is higher than the calculated dose. High-dose irradiation of the duodenum and stomach may cause serious gastrointestinal toxicity [9-11]. We should be more careful in performing high-precision radiation therapy. Therefore, we hypothesized that the dose distribution of RT planning for pancreatic cancer could be more reliably calculated by evaluating the relations of respiratory movement between the primary tumor and OARs.

The primary purpose of this study was to investigate the synchronization of respiration-induced motions at OARs for the primary tumor using 4DCT.

\section{Methods}

\section{Patients}

Patients with pancreatic cancer who underwent 4DCT at RT planning for three-dimensional conformal radiotherapy (3DCRT), intensity-modulated radiotherapy (IMRT) or stereotactic radiotherapy (SRT) between January 2015 and March 2016 was analyzed retrospectively.

\section{Computed tomography (CT) simulator}

Patients were immobilized in the supine position with both arms above their head. Intravenous contrast medium was administered after each patient had fasted for at least 3 hours. The slice thickness of the CT scan was $2 \mathrm{~mm}$. No fiducial markers were placed in the primary tumor. External abdominal compression was not delivered.

Two phases at the inhale and exhale phases in three-dimensional CT images and ten phases in 4DCT images using a 16-slice CT machine (Aquilion LB, Canon Medical Systems Corporation) and a real-time positioning management system (Varian Medical Systems, Palo Alto, CA) were acquired under the condition of shallow free breathing.

\section{Contouring}

RT planning was performed by ECLIPSE (Varian Medical Systems, Palo Alto, CA, USA). The gross tumor volume (GTV) and the duodenum (DU) and stomach (ST) were contoured in images at the 3D exhale phase. Then contouring of those images was adapted to $4 \mathrm{D}$ images of each phase using deformable image registration by MIM Maestro ${ }^{\text {TM }}$ software (ver. 6, MIM software, $\mathrm{OH}$, USA).

GTV was defined as the primary tumor identified on CT. The clinical target volume (CTV) was defined as GTV plus $5 \mathrm{~mm}$. Basically, the planning target volume (PTV) was defined as CTV plus 10-mm margins in 3DCRT. The PTV was defined as CTV plus $5 \mathrm{~mm}$ in IMRT or SRT. Contouring of the DU and ST was based on the report by Jabbour et al. [12]. The ST, separated into cardia, fundus, body, antrum and pylorus, was contoured as one organ. The DU was contoured as one organ from the first portion to the fourth portion. 


\section{Evaluation of movement in the contouring target}

First, we investigated the maximum respiratory movement in each contouring target. With reference to the $50 \%$ phase (exhale phase), the excursions of respiration-induced motions of the GTV, DU and ST at each phase were measured. The respiratory motion was calculated from the movement of each contouring target in the left-right (x-axis), anterior-posterior (y-axis), and cranio-caudal (z-axis) directions. Based on those distances, the 3D excursions of each contouring target were calculated. The center of each contouring target volume was used as the reference point in the measurement of 3D excursions. Differences in the maximum 3D excursions between the GTV and other contouring targets were assessed by the Mann-Whitney test.

Next, we examined the synchronization of respiration-induced motions. To investigate the synchronization of respiration-induced motions of other contouring targets for the GTV, we evaluated the changes in distances of the DU and ST in their positional relationships with the GTV for each respiratory phase. The differences between the synchronization of each contouring target in each respiratory phase was analyzed by the Kruskal-Wallis test. We defined no significant difference in this test as synchronization of respiratory movement between each target. We further examined the following methods to determine the reliability of this synchronization. First, we examined the differences in the overlap volumes between PTV and OARs in each respiratory phase. In evaluating the overlaps, a 10-mm margin of the planning organ at risk volume (PRV) was added to the stomach and duodenum. With reference to the $50 \%$ phase, the rates of change in the PTV overlap volumes in the DU and ST at each respiratory phase were measured. The differences of mean dose and maximum dose to the ST and DM in each respiratory phase were also evaluated. Similarly, the rates change in the mean dose and maximum dose was investigated based on the $50 \%$ phase. The differences between each respiratory phase in percentage changes of the PTV overlap volume, mean dose and maximum dose were also analyzed by the Kruskal-Wallis test.

Continuous variables are presented as mean values \pm standard deviation (SD). Statistical significance was set at the level of $p<0.05$. Statistical analysis was performed using JMP@10 (SAS Institute Inc., Cary, NC, USA).

\section{Results}

\section{Patient characteristics}

Nine patients with pancreatic cancer were analyzed in this study. Patient characteristics are shown in Table 1. Six patients had pancreas head/uncus cancer and 3 patients had body/tail cancer. The mean \pm SD of GTV volume was $24.50 \pm 8.75 \mathrm{cc}$. The mean \pm SD of PTV volume was $167.9 \pm 67.8 \mathrm{cc}$.

\section{Correlations between respiratory movements of the GTV and other contouring targets}


The distances for respiratory movements in the contouring targets are shown in Fig. 1. The means \pm SD of maximum excursions at the GTV were $2.0 \pm 0.9,3.2 \pm 1.3$ and $9.0 \pm 2.8 \mathrm{~mm}$ in the $x$-axis, $y$-axis, and zaxis directions, respectively. The means \pm SD of maximum 3D excursions at the GTV, DU and ST were 9.6 $\pm 3.3,9.8 \pm 4.6$ and $11.4 \pm 3.8 \mathrm{~mm}$, respectively.

The differences for respiratory movements between contouring targets are shown in Table 2. There was a significant difference in excursion in the $y$-axis direction between the GTV and ST $(p=0.034)$. There were no significant differences in maximum 3D excursions between GTV and ST/DU.

Table 1

Patient Characteristics

\begin{tabular}{|lllllll|}
\hline Patient & Age & Gender & $\begin{array}{l}\text { Tumor } \\
\text { location }\end{array}$ & $\begin{array}{l}\text { GTV volume } \\
\text { (cc) }\end{array}$ & $\begin{array}{l}\text { PTV volume } \\
\text { (cc) }\end{array}$ & $\begin{array}{l}\text { Treatment } \\
\text { technique }\end{array}$ \\
\hline 1 & 58 & Male & Uncus & 20.1 & 176.8 & 3DCRT \\
\hline 2 & 59 & Female & Head & 28.77 & 289.7 & 3DCRT \\
\hline 3 & 70 & Male & Uncus & 16.33 & 181.4 & 3DCRT \\
\hline 4 & 56 & Male & Uncus & 29.86 & 222.7 & 3DCRT \\
\hline 5 & 65 & Female & Body & 9.79 & 88.9 & 3DCRT \\
\hline 6 & 64 & Male & Body & 35.79 & 188.9 & 3DCRT \\
\hline 8 & 64 & Female & Head & 24.93 & 161 & 3DCRT \\
\hline 9 & 69 & Male & Body & 35.16 & 139.2 & SRT \\
\hline
\end{tabular}

Table 2

Results for maximum respiratory movement in each contouring target.

\begin{tabular}{|lllllllll|}
\hline & $\begin{array}{l}\text { 3D excursion } \\
(\mathrm{mm})\end{array}$ & $\begin{array}{l}\mathrm{P} \\
\text { value }\end{array}$ & $\begin{array}{l}\mathrm{X} \text {-axis } \\
(\mathrm{mm})\end{array}$ & $\begin{array}{l}\mathrm{P} \\
\text { value }\end{array}$ & $\begin{array}{l}\text { Y-axis } \\
(\mathrm{mm})\end{array}$ & $\begin{array}{l}\mathrm{P} \\
\text { value }\end{array}$ & $\begin{array}{l}\text { Z-axis } \\
(\mathrm{mm})\end{array}$ & $\begin{array}{l}\mathrm{P} \\
\text { value }\end{array}$ \\
\hline GTV & $9.6 \pm 3.3$ & ref & $\begin{array}{l}2.0 \pm \\
0.9\end{array}$ & ref & $\begin{array}{l}3.2 \pm \\
1.4\end{array}$ & ref & $9.0 \pm 3.0$ & ref \\
DU & $9.8 \pm 4.6$ & 0.627 & $\begin{array}{l}2.5 \pm \\
1.4\end{array}$ & 0.596 & $\begin{array}{l}3.5 \pm \\
1.6\end{array}$ & 0.508 & $9.2 \pm 4.4$ & 0.627 \\
& & & & & & & & \\
ST & $11.4 \pm 3.8$ & 0.31 & $\begin{array}{l}2.8 \pm \\
1.3\end{array}$ & 0.101 & $\begin{array}{l}4.6 \pm \\
1.3\end{array}$ & 0.034 & $\begin{array}{l}1.07 \pm \\
3.8\end{array}$ & 0.354 \\
\hline
\end{tabular}




\section{Synchronization of respiration-induced motions between the GTV and other contouring targets}

The results for synchronization of respiratory motions are shown in Fig. 2. At $0 \%$ and $90 \%$ phases (inhale phases), means \pm SD of distance changes in the positional relationship with the GTV were $0.3 \pm 1.9$ and $0.7 \pm 2.0 \mathrm{~mm}$, respectively, for the DM and $-2.5 \pm 1.7$ and $-2.4 \pm 2.0 \mathrm{~mm}$, respectively, for the ST. At $20 \%$ and $70 \%$ phases, those with the GTV were $0.5 \pm 0.7$ and $0.2 \pm 1.3 \mathrm{~mm}$, respectively, for the DU and $-0.9 \pm$ 0.8 and $-0.3 \pm 1.4 \mathrm{~mm}$, respectively for the ST. There was no significant difference in distance changes between each respiratory phase in the DU $(p=0.568)$ for GTV. The distance between the ST and GTV at the inhale phase was significantly shorter $(p<0.001)$.

The results for percentage change of PTV overlap in each respiratory phase are shown in Fig. 3. The means \pm SD of PTV overlap volumes at the $50 \%$ phase in the ST and DU were $20.39 \pm 21.82$ and $55.42 \pm$ $46.74 \mathrm{cc}$, respectively. At $0 \%$ and $90 \%$ phases, means \pm SD of percentage changes in PTV overlap volumes were $-1.7 \pm 7.5$ and $-3.0 \pm 9.8 \%$, respectively, for the DU and $6.3 \pm 11.9$ and $3.7 \pm 11.1 \%$, respectively for the ST. At $20 \%$ and $70 \%$ phases, means \pm SD of percentage changes in PTV overlap volumes were $-2.7 \pm 8.1$ and $-3.9 \pm 5.9 \%$, respectively, for the DM and $1.5 \pm 9.7$ and $1.4 \pm 6.3 \%$, respectively, for the ST. There was a slight increase in PTV overlap volume of the ST in the expiratory phase, but there were no significant differences between the respiratory phases either in ST or DU ( $p=$ 0.101 and 0.559$)$.

The results for percentage changes of mean doses and maximum doses of the ST and DU in each respiratory phase are shown in Fig. 4. The means \pm SD of mean doses at $50 \%$ phase in the ST and DU were $12.80 \pm 8.30$ and $25.57 \pm 14.81 \mathrm{~Gy}$, respectively. The means \pm SD of maximum doses at the $50 \%$ phase in the ST and DU were $47.79 \pm 6.90$ and $47.68 \pm 10.77 \mathrm{~Gy}$, respectively. At $0 \%$ and $90 \%$ phases, means \pm SD of percentage changes in mean doses were $-12.7 \pm 23.7$ and $-11.9 \pm 21.0 \%$, respectively, for the DU and $38.9 \pm 55.9$ and $33.8 \pm 51.7 \%$, respectively, for the ST, respectively. At $20 \%$ and $70 \%$ phases, means \pm SD of percentage changes in PTV overlap volumes were $-5.3 \pm 11.1$ and $-3.1 \pm 9.1$, respectively, for the DU and $20.5 \pm 28.1$ and $13.2 \pm 22.1 \%$, respectively, for the ST, respectively. There was a significant difference in the change rates of mean doses in the respiratory phases in ST $(p=0.023)$, but there was no significant difference in DU $(p=0.933)$. There were significant differences in the change rates of maximum doses in the respiratory phases in both the ST and DU ( $p=0.942$ and 0.297$)$.

\section{Discussion}

We have reported the relationships between the primary tumor and OARs due to respiratory movement in RT for pancreatic cancer. To the best of our knowledge, this is the first detailed report about the synchronization of respiratory movements. In the present study, we examined the absolute value of respiratory movement in the primary tumor itself, and our results are similar to previously reported results for 4DCT and cine magnetic resonance images $[6-8,13]$. In previous studies, the distances of the $x$-axis, $y$-axis and z-axis in respiratory movement were $0.7-4.9 \mathrm{~mm}, 2.0-6.5 \mathrm{~mm}$ and $5.2-13.4 \mathrm{~mm}$, respectively. 
Therefore, we think that the results for synchronization of respiratory movement in our study are reliable. We believe that an understanding of this synchronization would make it easier for radiation oncologists to set RT doses and fields in pancreatic cancer.

Regarding the maximum distance of respiratory movement at OARs, the difference in respiratory movements between the duodenum and primary tumor was not significant, while there was a significant difference in movements in the antero-posterior direction in the stomach and respiratory movement of the stomach was large overall. Although there are a few previous reports on respiratory movements of the stomach and duodenum, our results are similar to the results of those previous studies [14-17]. Watanabe et al. reported that intrafractional gastric motions were 11.7 $\pm 8.3,11.0 \pm 7.1,6.5 \pm 6.5,3.4 \pm 2.3$, $7.1 \pm 8.2$, and $6.6 \pm 5.8 \mathrm{~mm}$ for the superior, inferior, right, left, ventral and dorsal points, respectively [16]. Uchinami et al. reported that the average respiratory amplitudes of the stomach was $4.1 \pm 1.4,2.9 \pm 1.3$, and $10.1 \pm 4.5 \mathrm{~mm}$ in the anterior-posterior, left-right, and superior-inferior directions, respectively [17]. These results suggest that respiratory changes in the stomach and duodenum are as large as those in the primary lesion. The movements of the stomach and duodenum in the cranio-caudal direction were conspicuous as expected. Therefore, it seems necessary to consider the synchronization between GTV and the stomach/duodenum.

Regarding the synchronization of respiratory movements, it was found that there was no difference in the positional relationship between the duodenum and the primary tumor in each respiratory phase, but the distance between the stomach and primary tumor at the inspiratory phase was shortened. PTV overlap volume did not change significantly in the expiratory phase in both the ST and DU, but the mean dose to the ST clearly increased in the expiratory phase. As a result, it was found that the duodenum, but not the stomach, moved synchronously with the primary tumor and breathing. Taniguchi et al. reported how the respiratory phase impacts doses to normal organs during SRT for pancreatic cancer [18], and they demonstrated that the dose to the duodenum was higher in the inspiratory phase than in the expiratory phase and that there was a significant overlap of the PTV with the duodenum. Moreover, though there were no statistical differences in PTV overlap with the stomach, this overlap volume was increased at the inspiratory phase in their study. The results for the duodenum were different in their study and our study. Although there were differences in the number of cases, irradiation method, and PTV volume, the reason for the difference in the results is not clear. However, it is thought that the doses to the stomach and duodenum would be likely to change under the condition of free breathing. Changes in doses to the stomach and duodenum due to respiratory changes also occurred in SRT for hepatic cell carcinoma [19]. Therefore, a strict approach for respiratory movement may be required at dose escalation by SRT or IMRT. For example, Huguet et al. reported that gating around end-exhalation reduced pancreatic tumor motion by $46-60 \%$ [8]. In the cases undergoing IMRT or SRT in the present study, RT with gating was also performed. We consider that fiducial marker placement is also important as RT method against respiratory movement [20]. Taniguchi et al. reported that a large PTV volume produced more overlapping volume of the duodenum and stomach [18], and the PTV volumes was shown to be significantly correlated with the development of acute intestinal toxicity [21]. Therefore, those methods for respiratory movement would be necessary in the case of large PTV volume. 
There were some limitations in the present study. First, the number of cases in this study was small. Second, the distance between each target was from the center of the target in the present study, and evaluation of the target edge was not performed. However, we consider that this point would be supplemented by the results of PTV overlap and mean dose in the stomach and duodenum due to respiratory movement. Third, only 4DCT in RT planning was used in the analysis in our study, and variations of the intrafraction and interfraction during RT were not considered. Akimoto et al. showed that there was a change in the position of the pancreatic tumor during interfraction and intrafraction [22], and some studies have shown that 4DCT alone does not adequately reflect respiratory movement of pancreatic cancer during daily treatment $[23,24]$. Large deformation and displacement of the stomach and duodenum on CT images taken on separate days have also been reported [25,26]. Furthermore, there has been a report showing dose changes in the stomach and duodenum during interfraction [27]. Therefore, it seems that not only the technique for considering respiratory movement but also the setting of PRV margins for the duodenum and stomach is important. In fact, PRV has been established to determine dose constraints of the stomach and duodenum in RT for pancreatic cancer [28]. A more reproducible treatment plan must be made when performing high-dose irradiation for pancreatic cancer.

\section{Conclusions}

Our results showed a tendency for respiration-induced motions of the DU and ST for the GTV. The DU may shift synchronously with the GTV due to respiratory movement. The distance change of the ST in its positional relationship with the GTV was reduced in the inhale phase. There is a possibility that OARs are incidentally irradiated more than expected in RT for pancreatic cancer in a free breathing condition.

\section{Abbreviations}

3DCRT: Three-dimensional conformal radiotherapy; 4DCT: Four-dimensional computed tomography; CT: Computed tomography; CTV: Clinical target volume; DU: duodenum; GTV: Gross tumor volume; IMRT: Intensity-modulated radiation therapy; OARs: PRV: Planning organ at risk volume; Organs at risk; PTV: Planning target volume; RT: Radiotherapy; SD: standard deviation; SRT: Stereotactic radiation therapy; ST: Stomach

\section{Declarations}

\section{Acknowledgement}

The authors thank all of the patients who participated in the present study and all of the staff of the Department of Radiation Oncology in National Cancer Center Hospital for support of the present study.

\section{Authors' contributions}


$\mathrm{RU}, \mathrm{AW}, \mathrm{YK}, \mathrm{YI}$ and $\mathrm{JI}$ participated in the design of the study and reviewed the results. RU, $\mathrm{Yl}, \mathrm{KT}, \mathrm{KI}, \mathrm{NM}, \mathrm{HI}$ and $\mathrm{Jl}$ were responsible for the patient collection and performed radiation planning. RU, AW, YK, SN, HO, $\mathrm{NK}, \mathrm{HT}$ and $\mathrm{JI}$ were responsible for the statistical analysis. RU drafted the manuscript. AW, YI, KJ and JI helped to draft the manuscript. All authors read and approved the final manuscript.

\section{Funding}

No funding was received

\section{Availability of data and materials}

The datasets used and/or analyzed during the current study are available from the corresponding author on reasonable request.

\section{Ethics approval and consent to participate}

This study was approved by a local institutional review board (2016-058), and all the patients gave written informed consent before enrolment.

\section{Consent for publication}

All authors have given consent for publication.

\section{Competing interests}

The authors declare that they have no competing interests.

\section{Author details}

${ }^{1}$ Department of Radiation Oncology, National Cancer Center Hospital, Tokyo, Japan. ${ }^{2}$ Department of Radiation Oncology, Tohoku University Graduate School of Medicine, Sendai, Japan. ${ }^{3}$ Department of Radiation Oncology, Showa University School of Medicine, Tokyo, Japan.

\section{References}

1. Krishnan S, Chadha AS, Suh Y, Chen HC, Rao A, Das P, et al. Focal Radiation Therapy Dose Escalation Improves Overall Survival in Locally Advanced Pancreatic Cancer Patients Receiving Induction Chemotherapy and Consolidative Chemoradiation. Int J Radiat Oncol Biol Phys. 2016;94:755-65. 
2. Ben-Josef E, Schipper M, Francis IR, Hadley S, Ten-Haken R, Lawrence T, et al. A phase I/II trial of intensity modulated radiation (IMRT) dose escalation with concurrent fixed-dose rate gemcitabine (FDR-G) in patients with unresectable pancreatic cancer. Int J Radiat Oncol Biol Phys. 2012;84:116671.

3. Pollom EL, Alagappan M, von Eyben R, Kunz PL, Fisher GA, Ford JA, et al. Single- versus multifraction stereotactic body radiation therapy for pancreatic adenocarcinoma: outcomes and toxicity. Int $J$ Radiat Oncol Biol Phys. 2014;90:918-25.

4. Mahadevan A, Miksad R, Goldstein M, Sullivan R, Bullock A, Buchbinder E, et al. Induction gemcitabine and stereotactic body radiotherapy for locally advanced nonmetastatic pancreas cancer. Int J Radiat Oncol Biol Phys. 2011;81:e615-22.

5. Low DA, Nystrom M, Kalinin E, Parikh P, Dempsey JF, Bradley JD, et al. A method for the reconstruction of four-dimensional synchronized CT scans acquired during free breathing. Med Phys. 2003;30:1254-63.

6. Tai A, Liang Z, Erickson B, Li XA. Management of respiration-induced motion with 4-dimensional computed tomography (4DCT) for pancreas irradiation. Int J Radiat Oncol Biol Phys. 2013;86:90813.

7. Goldstein SD, Ford EC, Duhon M, McNutt T, Wong J, Herman JM. Use of respiratory-correlated fourdimensional computed tomography to determine acceptable treatment margins for locally advanced pancreatic adenocarcinoma. Int J Radiat Oncol Biol Phys. 2010;76:597-602.

8. Huguet F, Yorke ED, Davidson M, Zhang Z, Jackson A, Mageras GS, et al. Modeling pancreatic tumor motion using 4-dimensional computed tomography and surrogate markers. Int J Radiat Oncol Biol Phys. 2015;91:579-87.

9. Nakamura A, Shibuya K, Matsuo Y, Nakamura M, Shiinoki T, Mizowaki T, et al. Analysis of dosimetric parameters associated with acute gastrointestinal toxicity and upper gastrointestinal bleeding in locally advanced pancreatic cancer patients treated with gemcitabine-based concurrent chemoradiotherapy. Int J Radiat Oncol Biol Phys. 2012;84:369-75.

10. Cattaneo GM, Passoni P, Longobardi B, Slim N, Reni M, Cereda S et al. Dosimetric and clinical predictors of toxicity following combined chemotherapy and moderately hypofractionated rotational radiotherapy of locally advanced pancreatic adenocarcinoma. Radiother Oncol 2013; 108: 66-71. [9] Jabbour SK, Hashem SA, Bosch W, Kim TK, Finkelstein SE, Anderson BM, et al. Upper abdominal normal organ contouring guidelines and atlas: a Radiation Therapy Oncology Group consensus. Pract Radiat Oncol 2014; 4: 82-89.

11. Bae SH, Kim MS, Cho CK, Kang JK, Lee SY, Lee KN, et al. Predictor of severe gastroduodenal toxicity after stereotactic body radiotherapy for abdominopelvic malignancies. Int J Radiat Oncol Biol Phys. 2012;84:469-74.

12. Jabbour SK, Hashem SA, Bosch W, Kim TK, Finkelstein SE, Anderson BM, et al. Upper abdominal normal organ contouring guidelines and atlas: a Radiation Therapy Oncology Group consensus. Pract Radiat Oncol. 2014;4:82-9. 
13. Feng M, Balter JM, Normolle D, Adusumilli S, Cao Y, Chenevert TL, et al. Characterization of pancreatic tumor motion using cine MRI: surrogates for tumor position should be used with caution. Int J Radiat Oncol Biol Phys. 2009;74:884-91.

14. Hallman JL, Mori S, Sharp GC, Lu HM, Hong TS, Chen GT. A four-dimensional computed tomography analysis of multiorgan abdominal motion. Int J Radiat Oncol Biol Phys. 2012;83:435-41.

15. Heinzerling JH, Bland R, Mansour JC, Schwarz RE, Ramirez E, Ding C, et al. Dosimetric and motion analysis of margin-intensive therapy by stereotactic ablative radiotherapy for resectable pancreatic cancer. Radiat Oncol. 2011;6:146.

16. Watanabe $\mathrm{M}$, Isobe $\mathrm{K}$, Takisima $\mathrm{H}$, Uno $\mathrm{T}$, Ueno N, Kawakami H, et al. Intrafractional gastric motion and interfractional stomach deformity during radiation therapy. Radiother Oncol. 2008;87:425-31.

17. Uchinami Y, Suzuki R, Katoh N, Taguchi H, Yasda K, Miyamoto N, et al. Impact of organ motion on volumetric and dosimetric parameters in stomach lymphomas treated with intensity-modulated radiotherapy. J Appl Clin Med Phys. 2019;20:78-86.

18. Taniguchi CM, Murphy JD, Eclov N, Atwood TF, Kielar KN, Christman-Skieller C, et al. Dosimetric analysis of organs at risk during expiratory gating in stereotactic body radiation therapy for pancreatic cancer. Int J Radiat Oncol Biol Phys. 2013;85:1090-5.

19. Jung SH, Yoon SM, Park SH, Cho B, Park JW, Jung J, et al. Four-dimensional dose evaluation using deformable image registration in radiotherapy for liver cancer. Med Phys. 2013;40(1):011706.

20. van der Horst A, Lens E, Wognum S, de Jong R, van Hooft JE, van Tienhoven G, et al. Limited role for biliary stent as surrogate fiducial marker in pancreatic cancer: stent and intratumoral fiducials compared. Int J Radiat Oncol Biol Phys. 2014;89:641-8.

21. Ito $Y$, Okusaka T, Kagami Y, Ueno H, Ikeda M, Sumi M, et al. Evaluation of acute intestinal toxicity in relation to the volume of irradiated small bowel in patients treated with concurrent weekly gemcitabine and radiotherapy for locally advanced pancreatic cancer. Anticancer Res. 2006;26:3755-9.

22. Akimoto $M$, Nakamura $M$, Nakamura A, Mukumoto N, Kishi T, Goto $Y$, et al. Inter- and Intrafractional Variation in the 3-Dimensional Positions of Pancreatic Tumors Due to Respiration Under Real-Time Monitoring. Int J Radiat Oncol Biol Phys. 2017;98:1204-11.

23. Lens $E$, van der Horst A, Kroon PS, van Hooft JE, Davila Fajardo R, Fockens P, et al. Differences in respiratory-induced pancreatic tumor motion between $4 \mathrm{D}$ treatment planning $\mathrm{CT}$ and daily cone beam CT, measured using intratumoral fiducials. Acta Oncol. 2014;53:1257-64.

24. Ge J, Santanam L, Noel C, Parikh PJ. Planning 4-dimensional computed tomography (4DCT) cannot adequately represent daily intrafractional motion of abdominal tumors. Int $\mathrm{J}$ Radiat Oncol Biol Phys. 2013;85:999-1005.

25. Nakamura A, Shibuya K, Nakamura M, Matsuo Y, Shiinoki T, Nakata M, et al. Interfractional dose variations in the stomach and the bowels during breathhold intensity-modulated radiotherapy for pancreatic cancer: Implications for a dose-escalation strategy. Med Phys. 2013;40:021701. 
26. Liu F, Erickson B, Peng C, Li XA. Characterization and management of interfractional anatomic changes for pancreatic cancer radiotherapy. Int J Radiat Oncol Biol Phys. 2012;83:423-9.

27. Magallon-Baro A, Loi M, Milder MTW, Granton PK, Zolnay AG, Nuyttens JJ, et al. Modeling daily changes in organ-at-risk anatomy in a cohort of pancreatic cancer patients. Radiother Oncol. 2019;134:127-34.

28. Oar A, Lee M, Le H, Hruby G, Dalfsen R, Pryor D, et al. Australasian Gastrointestinal Trials Group (AGITG) and Trans-Tasman Radiation Oncology Group (TROG) Guidelines for Pancreatic Stereotactic Body Radiation Therapy (SBRT). Pract Radiat Oncol. 2020;10:136-46.

\section{Figures}

\section{(A) Gross tumor volume}

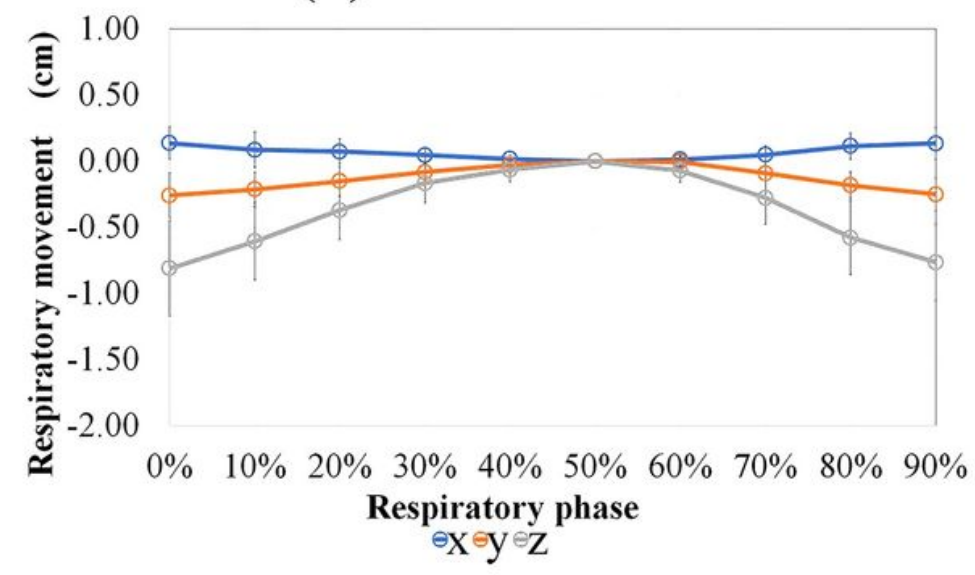

(B) Duodenum

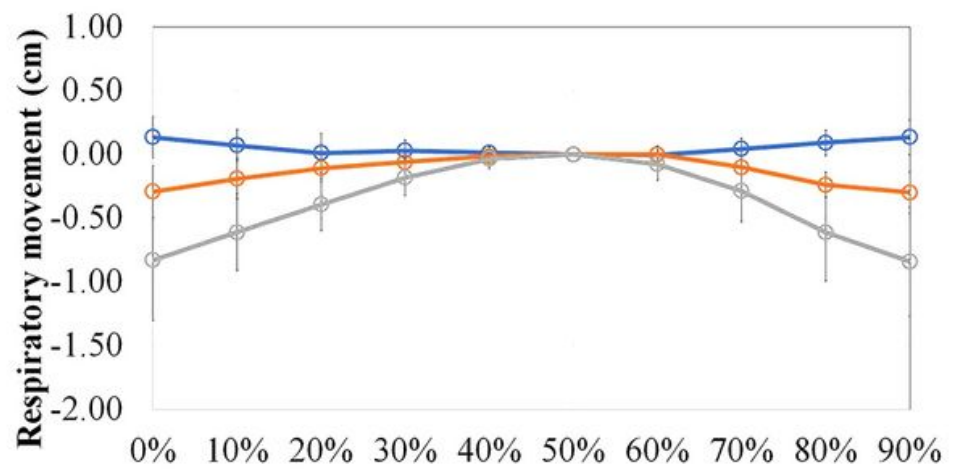

Respiratory phase

${ }^{\ominus} \mathrm{X} \ominus \mathrm{y} \bullet \mathrm{Z}$
(C) Stomach

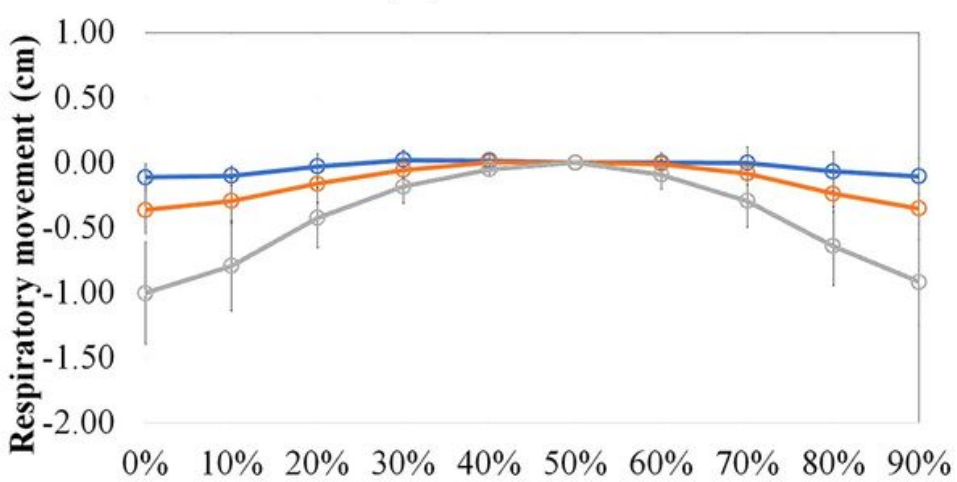

Respiratory phase

${ }^{\ominus} \boldsymbol{X}^{\ominus} \ominus^{\mathrm{Z}}$

(D) 3D excursion

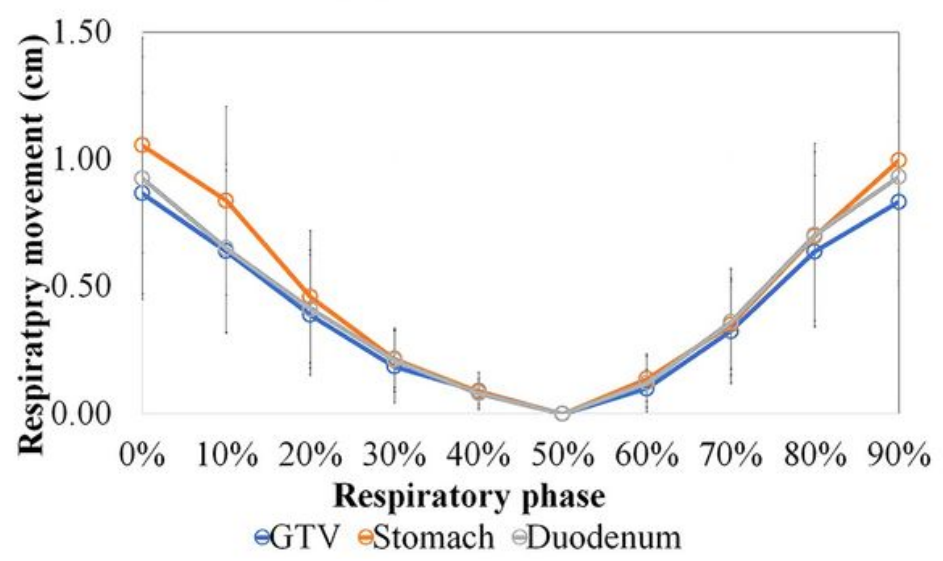

Figure 1

Results for movement distance at each respiratory phase in each target volume (A: gross tumor volume (GTV), B: duodenum, C: stomach, D: 3D excursion). 


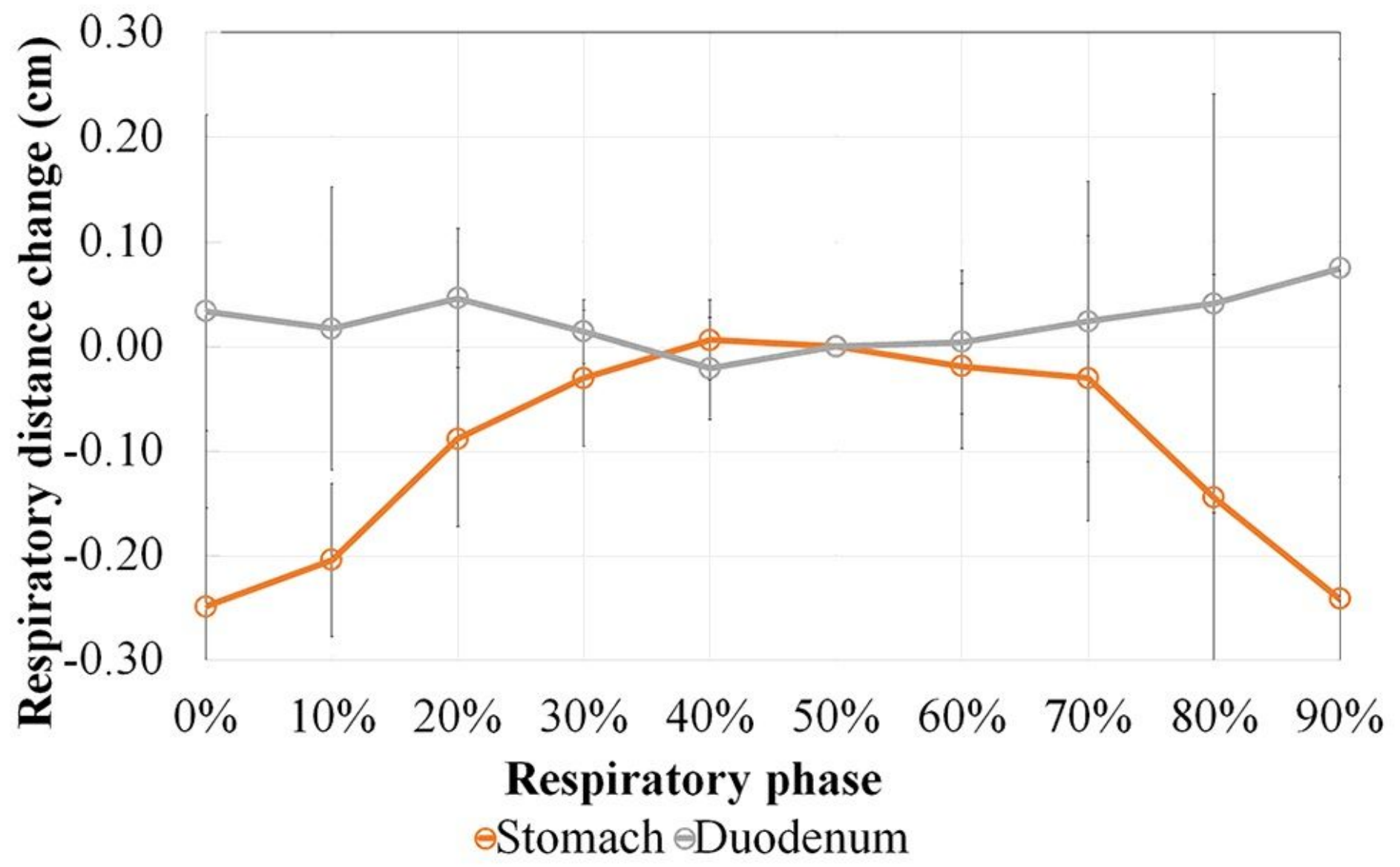

Figure 2

Results for synchronization by respiration-induced motion with organs at risk for the gross tumor volume. 


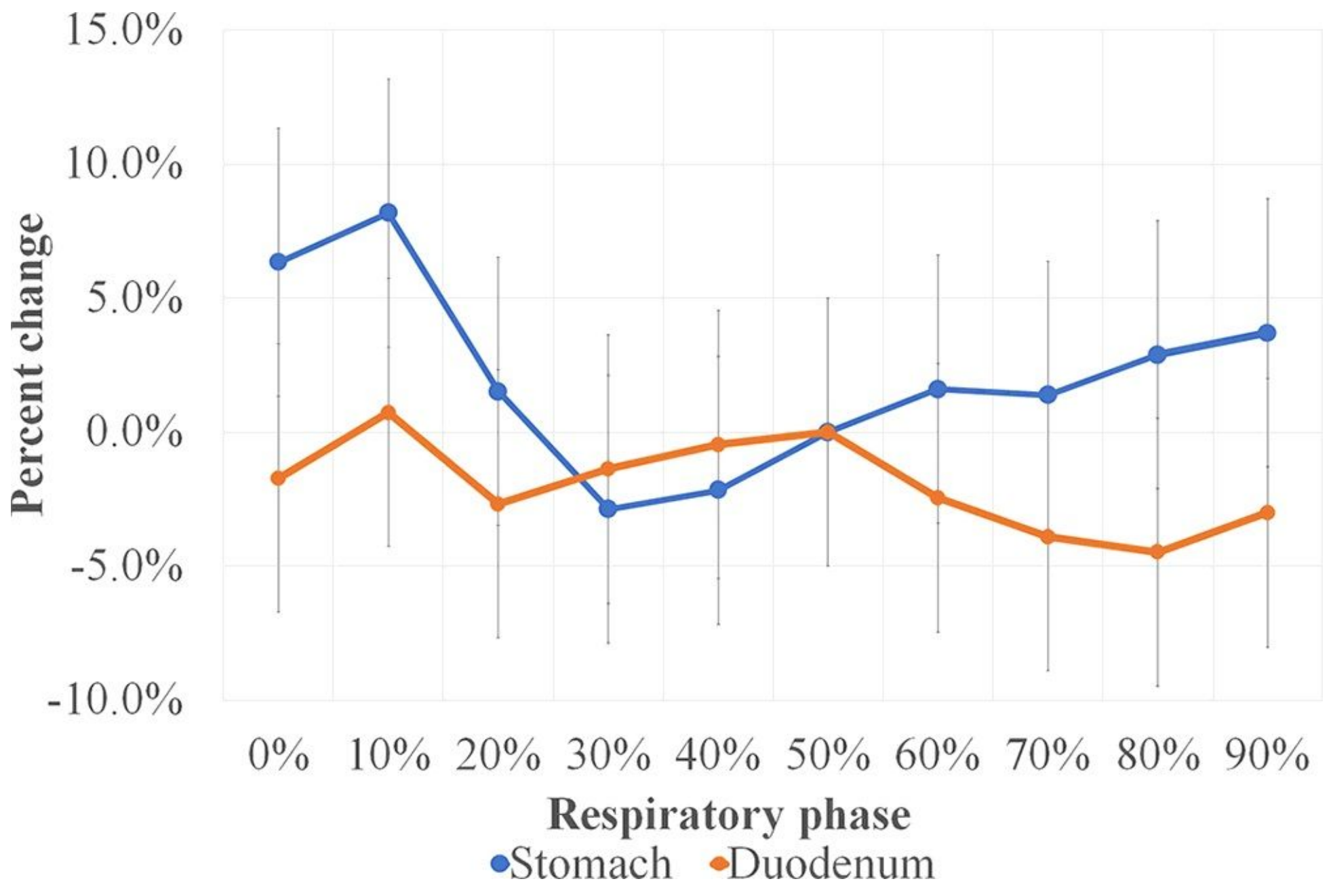

Figure 3

Results for differences in overlap volumes between planning target volume and stomach/ duodenum in each respiratory phase. 


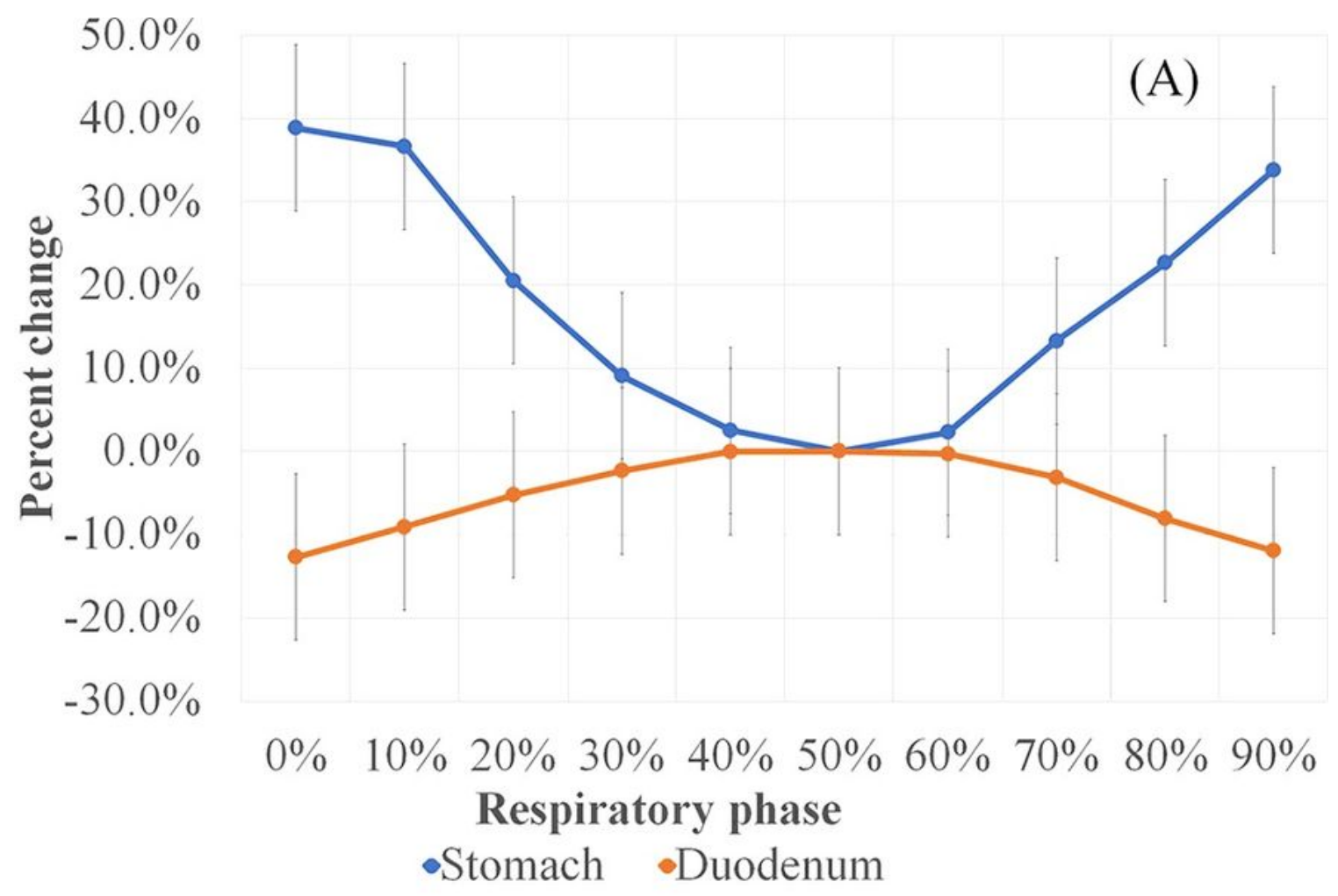

$40.0 \%$

$30.0 \%$

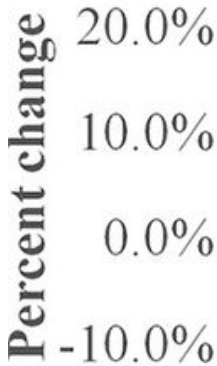

$-20.0 \%$

$-30.0 \%$

(B)

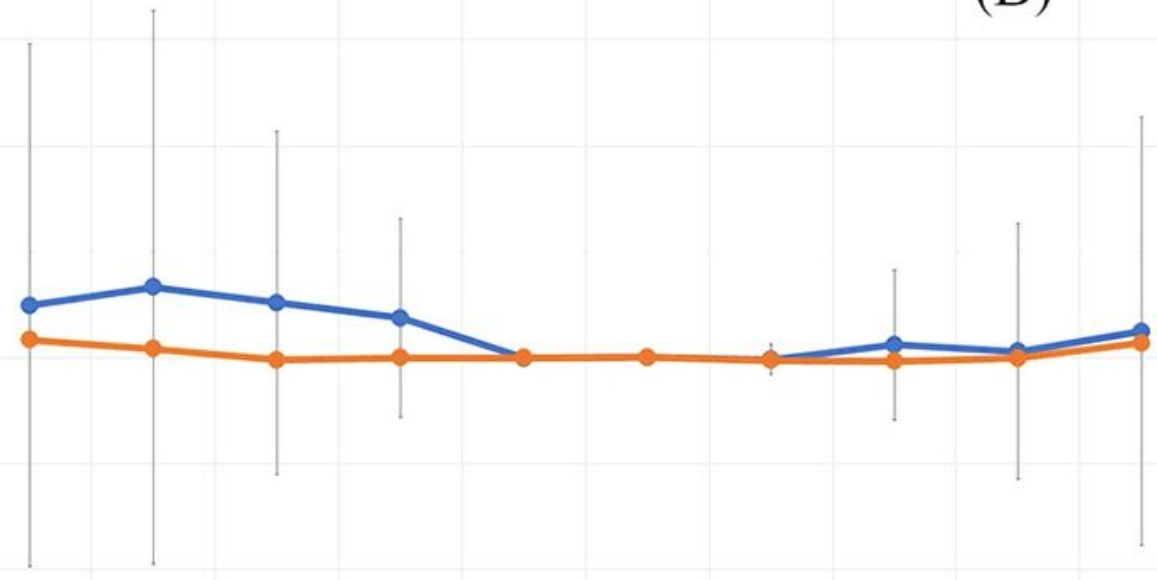

$\begin{array}{llllllllllllll}0 \% & 10 \% & 20 \% & 30 \% & 40 \% & 50 \% & 60 \% & 70 \% & 80 \% & 90 \%\end{array}$

Respiratory phase

-Stomach •Duodenum

\section{Figure 4}

Results for differences in mean doses $(A)$ and maximum doses $(B)$ to the stomach and duodenum in each respiratory phase. 